\title{
Positive and Negative Experiences of Social Support and Risk of Dementia in Later Life: An Investigation Using the English Longitudinal Study of Ageing
}

\author{
Mizanur Khondoker ${ }^{\mathrm{a}, \mathrm{b}, *}$, Snorri Bjorn Rafnsson ${ }^{\mathrm{c}, \mathrm{d}}$, Stephen Morris ${ }^{\mathrm{b}}$, Martin Orrell ${ }^{\mathrm{e}}$ \\ and Andrew Steptoe ${ }^{\mathrm{c}}$ \\ ${ }^{a}$ Norwich Medical School, University of East Anglia, Norwich, England, UK \\ ${ }^{\mathrm{b}}$ Department of Applied Health Research, University College London, London, UK \\ ${ }^{\mathrm{c}}$ Department of Epidemiology and Public Health, University College London, London, UK \\ ${ }^{\mathrm{d}}$ Centre for Primary Health and Social Care, London Metropolitan University, London, UK \\ ${ }^{\mathrm{e}}$ Institute of Mental Health, University of Nottingham, Nottingham, UK
}

Accepted 22 February 2017

\begin{abstract}
.
Background: Having a network of close relationships may reduce the risk of developing dementia. However, social exchange theory suggests that social interaction entails both rewards and costs. The effects of quality of close social relationships in later life on the risk of developing dementia are not well understood.

Objective: To investigate the effects of positive and negative experiences of social support within key relationships (spouse or partner, children, other immediate family, and friends) on the risk of developing dementia in later life.

Methods: We analyzed 10-year follow up data (2003/4 to 2012/13) in a cohort of 10,055 dementia free (at baseline) core participants aged 50 years and over from the English Longitudinal Study of Ageing (ELSA). Incidence of dementia was identified from participant or informant reported physician diagnosed dementia or overall score of informant-completed IQCODE questionnaire. Effects of positive and negative experiences of social support measured at baseline on risk of developing dementia were investigated using proportional hazards regression accommodating interval censoring of time-todementia.

Results: There were 340 (3.4\%) incident dementia cases during the follow-up. Positive social support from children significantly reduced the risk of dementia (hazard ratio, $\mathrm{HR}=0.83, p=0.042,95 \% \mathrm{CI}$ : 0.69 to 0.99 ). Negative support from other immediate family $(\mathrm{HR}=1.26, p=0.011, \mathrm{CI}$ : 1.05 to 1.50$)$; combined negative scores from spouse and children $(\mathrm{HR}=1.23$, $p=0.046$, CI: 1.004 to 1.51 ); spouse, children, and other family ( $\mathrm{HR}=1.27, p=0.021, \mathrm{CI}=1.04$ to 1.56$)$; other family $\&$ friends $(\mathrm{HR}=1.25, p=0.033, \mathrm{CI}: 1.02$ to 1.55$)$; and the overall negative scores ( $\mathrm{HR}=1.31, p=0.019, \mathrm{CI}: 1.05$ to 1.64$)$ all were significantly associated with increased risk of dementia.

Conclusion: Positive social support from children is associated with reduced risk of developing dementia whereas experiences of negative social support from children and other immediate family increase the risk. Further research is needed to better understand the causal mechanisms that drive these associations.
\end{abstract}

Keywords: Dementia, interval censoring, positive/negative social support, proportional hazards

${ }^{*}$ Correspondence to: Mizanur Khondoker, Norwich Medical School, University of East Anglia, Norwich, UK. Tel.: +44 1603 59 1501; E-mail: m.khondoker@uea.ac.uk. 


\section{INTRODUCTION}

Identifying ways to prevent or delay the onset of dementia is key to managing the health and economic impact of the disease. Recent research has demonstrated potential for primary prevention and delayed onset of the disease by manipulating exposure to modifiable risk factors [1]. Norton et al. [1] estimated that around a third of Alzheimer's disease cases worldwide might be attributable to potentially modifiable risk factors such as physical inactivity and diabetes. Most of the lifestyle and social factors are modifiable and there is substantial evidence in the literature suggesting a broad range of such factors that could influence the risk of cognitive decline and dementia [1-3].

A large body of literature exists that consistently shows that social connections have an important influence on health and wellbeing in older age $[4,5]$. For example, being more socially engaged $[6,7]$ and having a rich network of close relationships, including being married and having adult children [8-11], are thought to reduce the risk of cognitive decline and developing dementia. Specifically, older people's personal networks have been regarded as an important source of social support (e.g., assistance, perceived or actual, offered in the form of understanding and reassurance, financial help, personal advice, etc.) that enhances well-being and facilitates adaptation to life stress [12]. However, social interactions are thought to entail both rewards and costs [13], and research interest in the negative (being critical, unreliable, and annoying), as well as the positive (understanding, reliable, and approachable), aspects of personal social relations in older age has grown over the past decades [14]. A central focus of much of this research has been the question of which of the largely uncorrelated positive (e.g., enacted support including affect, encouragement, etc.) or negative (e.g., demands, criticism, etc.) dimensions of social support has greater impact on older persons' health and well-being [14, 15]. While positive and supportive social bonds can be satisfying and beneficial, negative social support can be a source of intense interpersonal stress which may have a negative impact on both physical and mental health of older adults [16]. Although there are published reports on the influence of positive and negative social support on disability [17], depressive symptoms [15], and psychological well-being [18, 19], to our knowledge, evidence on their potential impact on the risk of developing dementia in older age is lacking.
As part of the Promoting Independence in Dementia (PRIDE) research programme (http://www.ucl. ac.uk/psychiatry/pride/programme), the aim of this paper is to determine the relative importance of positive and negative experiences of social support in late life for subsequent development of dementia in a sample of participants from a large, prospective, population-based study of older people. Specifically, given that different members in older people's social networks may serve different roles and functions [15], we performed source-specific analyses whereby we studied the associations between positive and negative social support and dementia risk separately according to relationship type, i.e., spouse/partner, children, other immediate family, and friends. We hypothesized that: 1) positive social support would have a favorable influence on dementia incidence whereas negative support would increase the risk of developing dementia; and 2) these associations would be stronger (i.e., have larger effect sizes) for emotionally-close relationships such as spouses/partners and children relative to other family and friends.

\section{METHODS}

\section{Study sample}

We analyzed data on a cohort of 10,055 core participants who were dementia free in 2002/3 (wave 1) from ELSA [20], a panel study of a representative sample of men and women aged 50 and over living in the community in England. In addition to being dementia free at baseline, participants of the study sample were required to complete the questionnaire for positive or negative social support (Supplementary Table 1) for at least one of the relationships (spouse, children, other immediate family, friend). We refer "other immediate family" or "other family" to indicate family members not including children and spouse such as brothers, sisters, cousins, parents, or grandchildren . ELSA involves data collection by computer-assisted personal interviews every two years, and we identified dementia incidences from up to six waves, covering a period of ten years. The exposure variables (the experiences of positive and negative social support) were measured at baseline (wave 1).

\section{Ethical approval}

Ethical approval for all the ELSA waves was granted from the National Research and Ethics Committee (http://www.nres.npsa.nhs.uk/). 


\section{Identification of dementia incidence}

Information about dementia was reported either by the participant or an informant if an eligible participant was physically or cognitively impaired, in hospital or temporarily in care during the interview period. Any responsible adult (aged 16 years or over) who knew enough about the respondent's circumstances to be able to provide information about them, such as a close family member (partner or child), fulfilled the role of an informant. Incidence of dementia during the study period [wave 2 (2004) to wave 6(2012)] was identified from several sources of information: (i) participant or informant reported physician diagnosed dementia or Alzheimer's disease, or (ii) overall score of the informant completed IQCODE questionnaire. The short-form IQCODE questionnaire [21] consists of 16 items asking the informant to comment on the ability of the person compared with 10 years ago to perform various functions (e.g., remembering the names of family) on a 5 -point scale ranging from 1 (much improved) to 5 (much worse). We used the cut-off point of 3.5 of the overall score (average of the responses on all 16 items) to define dementia which has high specificity and good sensitivity[22]. On the basis of the above criteria, there were 340 incident cases of dementia within the study cohort during wave 2 (2004) to wave 6 (2012). Of the 340 cases, 284 were identified from the reports of physician diagnosed dementia or Alzheimer's disease and the remaining 56 were identified based on the IQCODE score.

\section{Exposures: Experience of positive and negative social support}

Measures of positive and negative experiences of social support for each relationship (spouse or partner, children, other immediate family, friends) were calculated based on responses at baseline (wave 1) on a set of six items within the self-completed "Health and lifestyle of people aged 50 and over" questionnaire. Three items were used to measure positive experiences of social support and the remaining three for negative experiences (see Supplementary Table 1). All items were measured on a 4-point scale ranging from 1 (at lot) to 4 (not at all). We reverse coded the scales so that higher value indicated more of the positive or negative experiences: 1 (not at all) to 4 (a lot). For each individual relationship (spouse, children, other family, or friend), experience of positive or negative support score was calculated by averag- ing the reverse coded scores of the three items (a, b and c) displayed in Supplementary Table 1. We have also calculated four sets of overall scores by averaging the scores for various combinations of individual relationships: (1) mean across all four relationships (spouse, children, other family, friends), (2) mean across spouse, children, and other family, (3) mean for spouse and children, and (4) mean for other family and friends. In calculating the mean scores from more than one relationship, we considered the mean of available (non-missing) data. Summary statistics (mean and standard deviations) of the overall scores as well those for individual relationships by gender are displayed in Table 1.

\section{Outcome measure: Time-to-dementia}

The outcome variable was defined as time-todementia from the start date of the ELSA study. As the follow-up interviews of the ELSA participants take place every two years, neither the time-to-dementia for incident cases nor the censoring time (due to drop-out/lost to follow-up or not developing dementia during the study period) was known exactly. Therefore, both the event time (time-to-dementia) and censoring time were treated as interval censored between two consecutive waves (a two-year interval) with an event indicator (1 for dementia incidence) distinguishing between dementia events and censoring. The participants who did not develop dementia by wave 6 were treated as censored at 120 months and were assigned within the final interval. For example, a participant reporting dementia at wave 3 (2006) who was dementia free at wave 2 (2004) was considered as having the disease at any time between 2004 and 2006 and was assigned the interval $(24,48]$ months as their time-to-event. We did not distinguish between losses to follow-up due to deaths and due to other reasons.

\section{Covariates}

We adjusted our analyses for potential confounding variables. We selected covariates for adjustment using both hypothesis and knowledge based approach as well as based on statistical assessment of confounding. The covariates age, sex, and net wealth, which are believed to have close links with cognition and family/social relationships [23-25], were included in all models. Net total wealth represented the sum of personal savings, investments, physical wealth, and housing wealth after financial debt 
Table 1

Summary statistics of covariates (age, net wealth) and positive and negative social support scores by gender

\begin{tabular}{lcc}
\hline Variable & Men & Women \\
\hline Size of the study sample: N (\%) & $5,475(54.45)$ & $4,580(45.55)$ \\
Age: Mean (SD) & $65.03(10.27)$ & $64.57(9.82)$ \\
Net wealth: Mean (SD) & $5.55(2.85)$ & $5.81(2.82)$ \\
& & \\
Positive social support scores: Mean (SD) & & $3.72(0.46)$ \\
Spouse $(n=7,075)$ & $3.56(0.60)$ & $3.32(0.71)$ \\
Children $(n=8,660)$ & $3.49(0.61)$ & $2.74(0.92)$ \\
Family $(n=8,826)$ & $2.94(0.92)$ & $2.95(0.74)$ \\
Friends $(n=9,173)$ & $3.29(0.71)$ & $3.51(0.55)$ \\
Spouse + Children $(n=9,270)$ & $3.52(0.55)$ & $3.24(0.59)$ \\
Spouse + Children + Family $(n=9,920)$ & $3.29(0.60)$ & $2.84(0.70)$ \\
Family + Friends $(n=9,684)$ & $3.11(0.67)$ & $3.17(0.52)$ \\
Overall (Spouse, Child, Family, Friend) $(n=10,055)$ & $3.30(0.51)$ & \\
& & $1.79(0.55)$ \\
Negative social support scores: Mean (SD) & & $1.68(0.61)$ \\
Spouse $(n=7,067)$ & $1.85(0.65)$ & $1.64(0.66)$ \\
Children $(n=8,649)$ & $1.62(0.60)$ & $1.61(0.55)$ \\
Family $(n=8,748)$ & $1.64(0.68)$ & $1.73(0.52)$ \\
Friends $(n=9,119)$ & $1.51(0.53)$ & $1.70(0.50)$ \\
Spouse + Children $(n=9,261)$ & $1.70(0.55)$ & $1.62(0.53)$ \\
Spouse + Children + Family $(n=9899)$ & $1.70(0.53)$ & $1.67(0.45)$ \\
Family + Friends $(n=9,636)$ & $1.57(0.52)$ & \\
Overall $($ Spouse, Child, Family, Friend) $(n=10,036)$ & $1.63(0.47)$ & \\
\hline Notes: 1 . Family implies immediate family members other than spouse or children. 2. Positive and negative support \\
scores were measured on a 4-point scale ranging from 1 (not at all) to $4($ a lot) so that higher value indicated more \\
of the positive or negative experiences. & & \\
& &
\end{tabular}

and mortgage debt have been subtracted, and is a key indicator of socioeconomic resources among older people [20]. We have also statistically assessed several other covariates including education and comorbidity conditions such as diabetes, cardiovascular disease, stroke, hypertension, and cancer for their role as potential confounders. Education was coded as a categorical variable with three levels (no education, primary/secondary education, and higher education). All the co-morbidity variables were defined as binary indicators (yes/no). Covariates passing statistical test for confounding effect, i.e., those were associated with both the exposure and outcome, were accounted for in the respective models in addition to age, sex, and net wealth.

\section{Statistical analysis}

Proportional hazards $(\mathrm{PH})$ regression models were used allowing for interval censoring of time-todementia for estimating and testing the effects of positive and negative social exchanges/support on the risk of developing dementia. Time-to-event or survival data are said to be interval censored when a subject's event time is not known exactly, but only that it lies between two values. Data of this type typically arise where follow-up is intermittent at fixed intervals. For the subjects who reported dementia at a follow-up (wave), all that is known is that time-to-dementia is at least as long as the time of the earlier follow-up and no longer than the time of the later follow-up. To accommodate this feature of the data in a PH regression model, we fitted the model by maximizing a transformed likelihood that enabled estimating the parameters of the $\mathrm{PH}$ model using standard statistical software (Stata). Hosmer et al. [26] (Chapter 7, pp. 231-243) showed that with some re-arrangement of data, the likelihood function for a $\mathrm{PH}$ survival regression model under interval censoring of events can equivalently be represented by the likelihood of a generalized linear model (GLM) of the event indicator (binary outcome) under binomial family and complementary log-log as the link (or linearizing) function. The amount of missing data on covariates were minimal with no missing data on age and sex, and only $1.5 \%$ missing data on net wealth. We therefore performed analysis of available data without any imputation of missing observations. However, measures on the exposure variable (positive or negative social support scores) 
Table 2

Hazard ratios (exponentiated coefficients), $p$-values, and 95\% confidence intervals for the Interval censored Proportional Hazard (PH) regression models. All models included the covariates age, sex and net wealth. The covariates education and diabetes were also controlled for in some models where they passed the tests for confounding. Statistically significant associations are shown in bold

\begin{tabular}{|c|c|c|c|c|c|c|}
\hline \multirow[t]{2}{*}{ Exposure variable (Score type) } & \multicolumn{3}{|c|}{ Positive scores } & \multicolumn{3}{|c|}{ Negative scores } \\
\hline & HR (SE) & $p$-value & $95 \% \mathrm{CI}$ & HR (SE) & $p$-value & $95 \% \mathrm{CI}$ \\
\hline Overall score & $0.87(0.09)$ & 0.171 & $(0.72,1.06)$ & $1.31(0.15)$ & 0.019 & $(1.05,1.64)$ \\
\hline Spouse + children score & $0.89(0.09)$ & 0.289 & $(0.73,1.10)$ & $1.23(0.13)$ & 0.046 & $(1.004,1.51)$ \\
\hline Spouse + children + Other family score & $0.93(0.08)$ & 0.450 & $(0.78,1.11)$ & $1.27(0.13)$ & 0.021 & $(1.04,1.56)$ \\
\hline Other family + friend score & $0.89(0.07)$ & 0.136 & $(0.76,1.04)$ & $1.25(0.13)$ & 0.033 & $(1.02,1.55)$ \\
\hline Spouse score & $0.83(0.09)$ & 0.107 & $(0.67,1.04)$ & $1.08(0.13)$ & 0.536 & $(0.85,1.35)$ \\
\hline Children score & $0.83(0.08)$ & 0.042 & $(0.69,0.99)$ & $1.19(0.12)$ & 0.075 & $(0.98,1.45)$ \\
\hline Other family score & $0.92(0.06)$ & 0.212 & $(0.81,1.05)$ & $1.26(0.11)$ & 0.011 & $(1.05,1.50)$ \\
\hline Friends score & $0.89(0.07)$ & 0.116 & $(0.76,1.03)$ & $1.14(0.12)$ & 0.238 & $(0.92,1.40)$ \\
\hline
\end{tabular}

for some individual relationships were not available for a substantial proportion of participants. For example, positive or negative support scores from spouse were not applicable for approximately $29 \%$ of the study participants who did not have a spouse or partner, and the corresponding analysis had to be based on a reduced sample. Number of subjects included in the $\mathrm{PH}$ regression analysis for each relationship is shown in the table of summary statistics (Table 1). Results of the primary analysis based on the above model are presented in Table 2.

\section{Sensitivity analysis}

Three sensitivity analyses were conducted in order to assess the robustness of the findings of the primary analysis. We hypothesized that the quality of relationships with spouse, children, other family, and friends influenced the risk of developing dementia in older age. Dementia, however, often takes a toll on social relationships, and so people who are very close to developing dementia may already show signs of cognitive problems, and experience deterioration of relationships. It is therefore possible that reverse causality partly explains the associations observed between quality of relationships and dementia. The first sensitivity analysis was designed to assess the robustness of the findings against possible reverse causality by excluding dementia cases diagnosed during the first two waves (i.e., 2 years) of the study.

The second sensitivity analysis was to assess robustness of the findings against the reliability of diagnosis of dementia by excluding cases diagnosed using the IQCODE, and limiting the analysis to physician diagnosed dementia or Alzheimer's disease only (284 incident cases).
The third sensitivity analysis compared the findings from the interval censored PH regression analysis with that of a standard Cox $\mathrm{PH}$ regression where the time-to-dementia was assumed to be known exactly. This is the most commonly used analysis for time-to-event data with standard censoring mechanism. Participants reporting dementia at wave 2 were assigned 24 months as their time to event, 48 months for those reporting at wave 3, 72 months at wave 4 , and so on. Subjects not developing dementia by wave 6 were treated as right censored at that point. Loss to follow-up due to death or for any other reason was also treated as right censored. Results for the sensitivity analyses are presented in Supplementary Tables 1-3.

\section{RESULTS}

\section{Main analysis}

The primary analysis included 10,055 dementiafree core participants at baseline (wave 1), with 5,475 (54\%) male and 4,580 (46\%) female subjects. There were 340 (3.4\%) incident cases of dementia during the study period (wave 2 to wave 6) of which 190 were male and 150 female cases. Actual number of participants included in individual regression models, however, varied between 7,067 and 10,055 with incident dementia cases varying between 199 and 340 , respectively, due to some missing data in positive or negative social support scores. The number was lowest for analyzing the spouse's score (7,075 for positive, and 7,067 for negative score) as this score is not applicable for participants without having a spouse or partner (see Table 1 for more details). 
Participants were aged 50 years and over with overall mean age 65 years and standard deviation 10 years. Overall, men were more likely (56\%) to have dementia than women (44\%). Participants with dementia were on average older at baseline (mean age $=76$ years for men and 74 years for women) than the participants without dementia (mean age $=65$ years for men and 64 years for women). In general, positive and negative support scores for each relationship were negatively correlated as expected (Pearson's correlation, $r=-0.50,-0.34,-0.28$, and -0.11 for spouse, children, other immediate family, and friend respectively). Correlation between positive and negative scores for cross-relationships (e.g., between positive scores for spouse and negative scores for children) were also negative, but weaker than those for within relationship correlation (varied from -0.02 to -0.15 ). Additional summary statistics of the covariates and positive and negative social support scores stratified by gender for individual relationships and their combinations are given in Table 1.

Results from the interval-censored $\mathrm{PH}$ regression analysis assessing the effects of positive and negative social support scores are displayed in Table 2. Of the positive social support measures, a higher score led to reduced estimated adjusted risk of dementia (estimated hazard ratio $<1$ ) for all the four relationships and their combinations (Table 2). An increase of one point in the positive social support score led to between $7 \%$ to $17 \%$ reduction in the risk of developing dementia (hazard ratio, HR, ranging between 0.83 and 0.93 ). However, only the effect of positive social support from children was found to be statistically significant at the $5 \%$ level $(\mathrm{HR}=0.83, p=0.042$, $95 \% \mathrm{CI}=0.69$ to 0.99 ). Effects of positive support scores from spouse $(\mathrm{HR}=0.83, p=0.107)$ showed a trend toward significance with the same effect size $(\mathrm{HR}=0.83)$ as that for children.

Negative social support measures, on the other hand, showed an opposite effect on the risk of developing dementia-overall, an increase in the magnitude of negative social support resulted in an elevated estimated risk (HR $>1)$ of dementia. Negative support scores showed stronger effects than the positive scores, leading to $8 \%$ to $31 \%$ increased risk of incident dementia (HR ranged between 1.08 and 1.31) for various relationships, although not all were statistically significant. Effects of negative experience of support from other immediate family $(\mathrm{HR}=1.26, p=0.011,95 \% \mathrm{CI}=1.05$ to 1.50$)$, combined negative scores from spouse and children
$(\mathrm{HR}=1.23, p=0.046,95 \% \mathrm{CI}=1.004$ to 1.51$), \mathrm{com}-$ bined negative scores from spouse, children, and other family ( $\mathrm{HR}=1.27, p=0.021,95 \% \mathrm{CI}=1.04$ to 1.56$)$, combined negative scores from other family and friends $(\mathrm{HR}=1.25, p=0.033,95 \% \mathrm{CI}=1.02$ to 1.55 ), and the overall negative scores (combined across spouse, children, other family, and friends) $(\mathrm{HR}=1.31, p=0.019,95 \% \mathrm{CI}=1.05$ to 1.64$)$ were all statistically significant at $5 \%$ level. Regression coefficients for the covariates age and net wealth were significantly associated with dementia in all models showing older and poorer at greater risk of dementia. Effects of education and diabetes were also statistically significant in some models with more education leading to lesser risk and presence of diabetes leading to higher risk of developing dementia. The effect of sex was not statistically significant in our study, although some studies [27] in the literature reported women to be at higher risk than men. Since higher incidence of dementia among women may be present primarily in those over 90 years [28], the younger ELSA cohort may explain why gender was not significant in our study. We, however, decided to keep sex in all models as the effect size was considerable in some of the models.

\section{Sensitivity analysis}

Sensitivity analysis excluding dementia cases within two years of baseline resulted in a smaller number of incident cases (292 dementia cases between 2006 and 2012) (see Supplementary Table 2). The reduced number of events resulted in a loss of statistical power (slightly inflated standard errors) and led to non-significant results for some of the effects found in the main analysis. However, the magnitude of the effects (hazard ratios) and direction of the associations remained similar to that of the main analysis. Several of the adjusted associations remained statistically significant despite the loss of statistical power.

The second sensitivity analysis excluded cases diagnosed using the IQCODE, and limited the analysis to physician diagnosed dementia or Alzheimer's disease only (results reported in Supplementary Table 3). This again resulted in a loss of events (incident cases of dementia ranged between 164 and 284 for different models) and statistical power. Analyses however led to the same conclusion that an increase in positive experience of social support reduces the risk of dementia (HRs ranged between 0.79 and 0.95 ) and that in negative experience increases the risk (HRs for 
each unit increase in negative score varied from 1.06 to 1.30 ).

The third sensitivity analysis using standard Cox $\mathrm{PH}$ regression model, which assumed timeto-dementia was known exactly and did not accommodate interval censoring of event times, produced almost identical results to that of the primary analysis taking account of interval censoring (Supplementary Table 4).

\section{DISCUSSION}

\section{Summary of main findings}

Our multivariable analysis demonstrated that, irrespective of the source (type of relationship) of social support, overall negative support was significantly associated with an increased risk dementia. Some measures of positive support significantly reduced the risk of dementia. More specifically, negative social support from spouse, children, and other immediate family increased the risk whereas positive support from children was associated with a reduced dementia risk. Effect sizes were generally larger for negative compared with positive social support. As expected, the magnitude of the favorable effects of positive support appeared greater for closer kinship (e.g., children compared with friends) but this pattern was less clear for negative support. Relatively stronger associations for the negative social support relative to the positive support may be indicative of the fact that stress of criticism and lack of reliability are possibly more harmful than the absence of a warm relationship. The robustness of these results was confirmed in a series of sensitivity analyses.

\section{Comparison with previous investigations}

To the best of our knowledge, this is the first study to investigate the influence of both positive and negative social support on incident dementia in community-residing older people. Therefore, a direct comparison of our results with previous studies was not possible. We, however, compared our findings with that from studies looking at the effects of positive and negative social support on general health and well-being of older people although such comparisons are still challenging due to important methodological differences across investigations. Our findings appear broadly consistent with a substantial literature documenting the disproportionate impact of negative social support on general health and well-being outcomes in older people [14]. For example, our results corroborate recent findings on the harmful impact of overall negative support on depressive symptoms [15], decline in activities of daily living and instrumental activities of daily living functioning [29], levels of psychological well-being and distress [16], and on disability [17]. In contrast, negative exchanges were not related to all-cause mortality in a sample middle-aged Dutch people [30]. Specifically, we demonstrated significant effects on dementia risk of negative interactions with both spouse and children when combined together but not when they were examined separately. In contrast, Okun and Keith [15] reported significant effects of negative support from spouses, but not children, on depressive symptoms in their analysis of older adults.

Despite the effect of overall positive social support not being statistically significant in our study, we found that positive exchanges with children offered protection against cognitive impairment. Overall, this supports some previous studies on depressive symptoms [15] and positive well-being in older people [16]. Our results corroborate those from some studies [15] who reported that positive support from children, in particular, was associated with less depression symptoms in older people. In addition, they also found protective, albeit weaker, effects of positive exchanges with spouses and other family and friends. We observed a similar pattern with reasonably large effect sizes although our results did not pass the conventional 5\% significance threshold for spouse, other family and friend's scores.

\section{Possible mechanisms}

If indeed causal, the association between positive and negative social support and development of dementia may involve several different processes which, independently or in combination, may induce cognitive impairment in older people. For example, lack of supportive social relationships may promote health-damaging behaviors including smoking, alcohol drinking, sedentary lifestyle, poor dietary choices, and excess body weight [30]. These findings are important in light of the extensive research available documenting the adverse influences of smoking and physical inactivity on both cardiovascular [31] and cognitive outcomes [32] in older adults. Similarly, diverse psychosocial processes, including personality traits or individual differences, emotions and moods, and perceptions 
of personal control, may enhance people's coping strategies and adaptation to interpersonal stressors [12]. Importantly, individual's cognitive appraisal of the significance of an event or circumstances may mediate the impact of stressors on physical and mental health [16]. In turn, the effects of these psychological factors and states may be mediated through diverse psychobiological processes, including neuroendocrine and inflammatory responses $[33,34]$.

\section{Potential limitations}

First, due to being a relatively younger (age 50 years+) cohort and dementia case status being based primarily on self-reported doctor's diagnosis the incidence of dementia in our study was lower (3.4\%) than the incidence reported in other analyses such as [35] which reported an estimate of $6.5 \%$ overall in older people. Self-reported diagnoses may also have led to under reporting of milder cases. Second, further loss of cases has occurred due to exposure data (positive or negative social support scores) not being available for some participants leading to reduced sample size for some analyses. This was particularly the case for the analyses involving spouses' support scores as they are not applicable for people without a spouse or partner. We performed analysis of available data as imputation would not be appropriate given the nature of much of the missing data (e.g., support score from spouse in people not having a spouse, or from children in people not having children). However, despite the possibility of missing cases, we believe this is unlikely to induce major bias in our results given the consistency with other similar studies that used more objective evaluations of dementia [36, 37]. Although we were unable to verify the accuracy of the diagnostic information, self-reports of other conditions, including stroke [38], correspond highly with physician diagnoses, even in the presence of overt impaired cognition.

Third, it was not possible to consider the potential impact of genetic factors such as family history in this study as ELSA does not collect this information. Also, precise information about the type of dementia diagnosed was not available. However, there should not be many cases of familial dementia as relatively few cases of dementia were reported in participants less than 65 years.

Finally, given that negative support from other immediate family was found to increase the risk of dementia, it would be interesting to look at the asso- ciation of individual relationship within the other immediate family category with dementia. However, it was not possible to measure positive/negative social support scores for individual relationships within the other immediate family from the ELSA study.

\section{Conclusions}

In this investigation of older participants in the ELSA study, positive social support from children in later life was associated with a lower risk of developing dementia. Negative social support in a range of relationships, on the other hand, was associated with an increased risk of developing dementia. This work is a step toward better understanding of impact of social relationships on dementia risk; however, further research is needed to better establish any potential causal mechanisms that may drive these associations particularly with reference to age related social factors. Our findings add to the growing evidence on the relevance of social relationships for cognitive health in older age. Specifically, for health and social care practice, they highlight the value of addressing social relationship issues in individuals vulnerable to dementia, while pointing towards specific ways of potentially modifying risk. Similarly, at the policy level, our results will add to the impetus underlying local and national efforts to ameliorate the psychosocial conditions of older people.

\section{ACKNOWLEDGMENTS}

The PRIDE study was funded by the UK Economic and Social Research Council (ESRC) (Grant ES/L001802/1). The English Longitudinal Study of Ageing is funded by the National Institute on Aging (Grants 2RO1AG7644-01A1 and 2RO1AG017644) and by a consortium of UK government departments coordinated by the ESRC. The data are lodged with the UK Data Archive. Andrew Steptoe is supported by the British Heart Foundation. The funders of this study had no role in the study design, data collection, data analysis, data interpretation, or writing of the report.

Authors' disclosures available online (http://j-alz. com/manuscript-disclosures/16-1160r1).

\section{SUPPLEMENTARY MATERIAL}

The supplementary material is available in the electronic version of this article: http://dx.doi.org/ 10.3233/JAD-161160. 


\section{REFERENCES}

[1] Norton S, Matthews FE, Barnes DE, Yaffe K, Brayne C (2014) Potential for primary prevention of Alzheimer's disease: An analysis of population-based data. Lancet Neurol 13, 788-794. Erratum in Lancet Neurol 13, 1070.

[2] Hendrie HC (1998) Epidemiology of dementia and Alzheimer's disease. Am J Geriatr Psychiatry 6, S3-18.

[3] Prince M, Albanese E, Guerchet M, Prina M (2014) World Alzheimer Report 2014. Dementia and risk reduction: An analysis of protective and modifiable factors. Alzheimer's Disease International, London, Available from: https://www.alz.co.uk/research/world-report-2014.

[4] Cohen S, Janicki-Deverts D (2009) Can we improve our physical health by altering our social networks? Perspect Psychol Sci 4, 375-378.

[5] Thoits PA (2011) Mechanisms linking social ties and support to physical and mental health. J Health Soc Behav 52, 145-161.

[6] Marioni RE, Proust-Lima C, Amieva H, Brayne C, Matthews FE, Dartigues JF, Jacqmin-Gadda H (2015) Social activity, cognitive decline and dementia risk: A 20year prospective cohort study. BMC Public Health 15, 1089.

[7] Shankar A, Hamer M, McMunn A, Steptoe A (2013) Social isolation and loneliness: Relationships with cognitive function during 4 years of follow-up in the English Longitudinal Study of Ageing. Psychosom Med 75, 161-170.

[8] Fan LY, Sun Y, Lee HJ, Yang SC, Chen TF, Lin KN, Lin CC, Wang PN, Tang LY, Chiu MJ (2015) Marital status, lifestyle and dementia: A nationwide survey in Taiwan. PLoS One 10, e0139154.

[9] Feng L, Ng XT, Yap P, Li J, Lee TS, Hakansson K, Kua EH, Ng TP (2014) Marital status and cognitive impairment among community-dwelling chinese older adults: The role of gender and social engagement. Dement Geriatr Cogn Dis Extra 4, 375-384.

[10] Sundstrom A, Westerlund O, Kotyrlo E (2016) Marital status and risk of dementia: A nationwide populationbased prospective study from Sweden. BMJ Open 6, e008565.

[11] Sundstrom A, Westerlund O, Mousavi-Nasab H, Adolfsson R, Nilsson LG (2014) The relationship between marital and parental status and the risk of dementia. Int Psychogeriatr 26, 749-757.

[12] Uchino BN (2006) Social support and health: A review of physiological processes potentially underlying links to disease outcomes. J Behav Med 29, 377-387.

[13] Homans GC (1958) Social behavior as exchange. Am $J$ Sociol 63, 597-606.

[14] Rook KS (1997) Positive and negative social exchanges: Weighing their effects in later life. J Gerontol B Psychol Sci Soc Sci 52, S167-S169.

[15] Okun MA, Keith VM (1998) Effects of positive and negative social exchanges with various sources on depressive symptoms in younger and older adults. J Gerontol B Psychol Sci Soc Sci 53B, P4-P20.

[16] Newsom JT, Rook KS, Nishishiba M, Sorkin DH, Mahan TL (2005) Understanding the relative importance of positive and negative social exchanges: Examining specific domains and appraisals. J Gerontol B Psychol Sci Soc Sci 60, P304P312.

[17] Mavandadi S, Rook KS, Newsom JT (2007) Positive and negative social exchanges and disability in later life: An investigation of trajectories of change. J Gerontol B Psychol Sci Soc Sci 62, S361-S370.

[18] Ha J-H (2010) The effects of positive and negative support from children on widowed older adults' psychological adjustment: A longitudinal analysis. Gerontologist 50, 471481.

[19] Rook KS (1984) The negative side of social interaction: Impact on psychological well-being. J Pers Soc Psychol 46, 1097-1108.

[20] Steptoe A, Breeze E, Banks J, Nazroo J (2013) Cohort profile: The English longitudinal study of ageing. Int J Epidemiol 42, 1640-1648.

[21] Jorm A (1994) A short form of the Informant Questionnaire on Cognitive Decline in the Elderly (IQCODE): Development and cross-validation. Psychol Med 24, 145-153.

[22] Quinn TJ, Fearon P, Noel-Storr AH, Young C, McShane R, Stott DJ (2014) Informant Questionnaire on Cognitive Decline in the Elderly (IQCODE) for the diagnosis of dementia within community dwelling populations. Cochrane Database Syst Rev, CD010079.

[23] Kensinger EA, Corkin S (2009) Cognition in aging and age related disease. In Handbook of the Neuroscience of Aging, Hof PR, Mobbs CV, eds. Elsevier/Academic Press, Amsterdam/Boston, pp. 249-256.

[24] Gur RE, Gur RC (2002) Gender differences in aging: Cognition, emotions, and neuroimaging studies. Dialogues Clin Neurosci 4, 197-210.

[25] Vohs KD, Mead NL, Goode MR (2006) The psychological consequences of money. Science 314, 1154-1156.

[26] Hosmer DW, Lemeshow S, May S (2008) Applied Survival Analysis: Regression Modelling of Time to Event Data, John Wiley \& Sons, Inc.

[27] Andersen K, Launer LJ, Dewey ME, Letenneur L, Ott A, Copeland JR, Dartigues JF, Kragh-Sorensen P, Baldereschi M, Brayne C, Lobo A, Martinez-Lage JM, Stijnen T, Hofman A (1999) Gender differences in the incidence of $\mathrm{AD}$ and vascular dementia: The EURODEM Studies. EURODEM Incidence Research Group. Neurology 53, 1992-1997.

[28] Ruitenberg A, Ott A, van Swieten JC, Hofman A, Breteler MM (2001) Incidence of dementia: Does gender make a difference? Neurobiol Aging 22, 575-580.

[29] Krause N, Shaw BA (2002) Negative interaction and changes in functional disability during late life. $J$ Soc Pers Relat 19, 339-359.

[30] Croezen S, Picavet HSJ, Haveman-Nies A, Verschuren WMM, de Groot LC, van't Veer P (2012) Do positive or negative experiences of social support relate to current and future health? Results from the Doetinchem Cohort Study. BMC Public Health 12, 65.

[31] Kromhout D, Menotti A, Kesteloot H, Sans S (2002) Prevention of coronary heart disease by diet and lifestyle. Circulation 105, 893-898.

[32] Deary IJ, Corley J, Gow AJ, Harris SE, Houlihan LM, Marioni RE, Penke L, Rafnsson SB, Starr JM (2009) Age-associated cognitive decline. Br Med Bull 92, 135-152.

[33] Kiecolt-Glaser JK, Gouin J-P, Hantsoo L (2010) Close relationships, inflammation, and health. Neurosci Biobehav Rev 35, 33-38.

[34] Laura DK, Teresa ES, Glymour MM (2014) Biological pathways linking in social conditions and health: Plausible mechanisms and emerging puzzles. In Social Epidemiology, 2nd edition, Berkman LF, Kawachi I, Glymour MM, eds. Oxford University Press, Oxford, UK. 
[35] Matthews FE, Arthur A, Barnes LE, Bond J, Jagger C, Robinson L, Brayne C, Function MRCC, Collaboration A (2013) A two-decade comparison of prevalence of dementia in individuals aged 65 years and older from three geographical areas of England: Results of the Cognitive Function and Ageing Study I and II. Lancet 382, 1405-1412.

[36] Holwerda TJ, Deeg DJH, Beekman ATF, van Tilburg TG, Stek ML, Jonker C, Schoevers RA (2014) Feelings of loneliness, but not social isolation, predict dementia onset: Results from the Amsterdam Study of the Elderly (AMSTEL). J Neurol Neurosurg Psychiatry 85, 135-142.
[37] Wilson RS, Krueger KR, Arnold SE, Schneider JA, Kelly JF, Barnes LL, Tang Y, Bennett DA (2007) Loneliness and risk of alzheimer disease. Arch Gen Psychiatry 64, 234-240.

[38] Jin Y-P, Di Legge S, Østbye T, Feightner JW, Saposnik G, Hachinski V (2010) Is stroke history reliably reported by elderly with cognitive impairment? A community-based study. Neuroepidemiology 35, 215-220. 